DOI: $10.26730 / 1999-4125-2019-3-75-81$

УДК 622.831

РАСЧЕТ КРЕПИ С УЧЕТОМ ТАМПОНАЖА ЗАКРЕПНОГО ПРОСТРАНСТВА

\title{
CALCULATION OF THE SUPPORT WITH FILLING OF THE SPACE BEHIND THE SUPPORT
}

Тациенко Виктор Прокопьевич, доктор техн. наук, e-mail: ipeb@kuzstu.ru Victor P. Tatsienko, Dr. Sc. in Engineering

Гоголин Вячеслав Анатольевич, доктор техн наук, професcop, e-mail: gva.pv@,kuzstu.ru

Vyacheslav A. Gogolin, Dr. Sc. in Engineering

Ермакова Инна Алексеевна, доктор техн наук, профессор, e-mail: eia.pm $a$,kuzstu.ru Inna A. Ermakova, Dr. Sc. in Engineering

Лесин Юрий Васильевич, доктор техн наук, профессор,

Yury V. Lesin, Dr. Sc. in Engineering

Лисковец Александр Сергеевич, старший научный сотрудник, e-mail: promsnab kmramail.ru

Alexander S. Liskovets, senior research scientist

Кузбасский государственный технический университет имени Т.Ф. Горбачева, 650000, Россия, г. Кемерово, ул. Весенняя, 28

T.F. Gorbachev Kuzbass State Technical University, 28 Vesennyaya street, Kemerovo, 650000, Russian Federation

\section{Аннотация:}

В работе впервые рассчитывается напряженно-деформированное состояние системы «крепь-тампонажный слой -массив горных пород». Поведение массива горных пород описывается с учетом образования зоны нарушенных горных пород. Использован критерий прочности горных пород при объемном сжатии, который позволил выделить зону нарушенных горных пород. Приведено уравнение гиперболической зависимости окружных напряжений и деформачий в зоне нарушенных пород. С учетом пропорииональности окружных и радиальных напряжений в этой зоне получено решение уравнений равновесия для этих напряжений. С учетом непрерывности радиальных смещений и радиальных напряжений на границах кольчевых блоков «крепь - тампонажный слой - наруиенные породы-массив ненарущенных пород» получены уравнения для расчета давления на крепь и смещений верхняка. Проверяется прочность крепи по критерию Мора и прочность тампонажного слоя по критерию разруиения при объемном сжатии. Приводится критерий образования зоны нарушенных горных пород, учитывающий глубину разработки и прочность пород на сжатие. Разработанная методика позволяет рассчитать давление на крепь и смещение верхняка в зависимости от иирины тампонажного слоя и его модуля деформаций.

Ключевые слова: крепь, тампонажный слой, давление на крепь, смещение верхняка.

Abstract:

In the article, the calculation of the stress-strain state of the system «support-filling layer - rock mass» is shown for the first time. The behavior of the rock mass is described taking into account the formation of a zone of disturbed rocks. The strength criterion of rocks with bulk compression is used to highlight the zone of disturbed 
rocks. The equation of the hyperbolic dependence between circumferential stresses and deformations in the zone of disturbed rocks is given. The solution of the equilibrium equations is obtained taking into account the proportionality of the circumferential and radial stresses in this zone. The equations for calculating the pressure on the lining and the displacements of the upper member were obtained taking into account the continuity of radial displacements and radial stresses at the boundaries of the ring blocks «support-filling layer - disturbed rocks - an array of undisturbed rocks». The strength of the support is checked by the criterion of More, the strength of the filling layer is checked by the criterion of failure under bulk compression. The criterion for the formation of a zone of disturbed rocks has been developed and takes into account the depth of development and the compressive strength of rocks. The developed method allows to calculate the pressure on the support and displacement of the roof girder depending on the width of the filling layer and its deformation modulus.

Key words: support, filling layer, pressure on support, displacement of the roof girder.

Проблема расчета рамных крепей в настоящее время не является полностью решенной. Для решения даной проблемы использовались различные методы: инженерные эмпирические методы и методы строительной механики [1-4], аналитические методы теории упругости $[5,6]$, численные методы геомеханики $[7,8]$. В работах $[9,10]$ сделана попытка оценки несущей способности крепи с тампонажем закрепного пространства. Общие подходы к крешению проблемы расчета крепи изложены в [11-14]. В данной работе предлагается комплесный подход к расчету крепи с тампонажем закрепного пространства, учитывающий результаты стендовых испытаний крепей, численное моделирование этих испытаний [15] и аналитические расчеты взаимодействия геомеханической системы «крепь тампонажный слой - слой нарушенных пород массив ненарушенных горных пород».

\section{1. Напряженно-деформированное состояние крепи}

Распределение радиальных и окружных напряжений крепи получено в работе [15] и имеет следующий вид:

$$
\left\{\begin{array}{l}
\sigma_{r}(r)=\frac{q \cdot R_{1}^{2}}{R_{1}^{2}-R^{2}}-\frac{R^{2} \cdot R_{1}^{2} \cdot q}{R_{1}^{2}-R^{2}} \cdot \frac{1}{r^{2}} \\
\sigma_{\theta}(r)=\frac{q \cdot R_{1}^{2}}{R_{1}^{2}-R^{2}}+\frac{R^{2} \cdot R_{1}^{2} \cdot q}{R_{1}^{2}-R^{2}} \cdot \frac{1}{r^{2}} .
\end{array}\right.
$$

Здесь $R, R_{1}$ - внутренний и внешний радиус крепи, $r$ - расстояние от центра выработки, $q$ - давление на крепь со стороны тампонажного слоя.

Напряжения на внутренней поверхности крепи при $r=R$ находятся из выражений (1).

$$
\left\{\begin{array}{c}
\sigma_{r}(R)=0 \\
\sigma_{\theta}(R)=\frac{2 \cdot q \cdot R_{1}^{2}}{R_{1}^{2}-R^{2}} .
\end{array}\right.
$$

Смешение (прогиб) верхняка крепи вычисляется как

$$
u(R)=\frac{1}{E_{k}} \cdot \frac{q \cdot R^{2}}{h_{k}},
$$

где $E_{\mathrm{k}}-$ модуль деформации материала крепи, $h_{\mathrm{k}}$ - высота профиля крепи.

\section{2. Напряженио-деформированшое состояние тампонажного слоя}

Рапределение напряжений в тампонажном слое описывается выражениями (1) с учетом следующих граничных условий.

Давление на тампонажный слой со стороны крепи равно давлению на крепь $p_{1}=q$, давление на тампонажный слой со стороны нарушенных пород равно $p_{t}$.

Тогда уравнения (1) принимают следующий вид:

$$
\left\{\begin{array}{l}
\sigma_{r}(r)=\frac{p_{t} \cdot R_{t}^{2}-q \cdot R_{1}^{2}}{R_{t}^{2}-R_{1}^{2}}-\frac{R_{t}^{2} \cdot R_{1}^{2} \cdot\left(p_{t}-q\right)}{R_{t}^{2}-R_{1}^{2}} \cdot \frac{1}{r^{2}} ; \\
\sigma_{\theta}(r)=\frac{p_{t} \cdot R_{t}^{2}-q \cdot R_{1}^{2}}{R_{t}^{2}-R_{1}^{2}}+\frac{R_{t}^{2} \cdot R_{1}^{2} \cdot\left(p_{t}-q\right)}{R_{t}^{2}-R_{1}^{2}} \cdot \frac{1}{r^{2}},
\end{array}\right.
$$

Здесь $R_{1}$ и $R_{t}-$ внутренний и внешний радиус тампонажного слоя.

Напряжения на внутренней поверхности тампонажного слоя определяются из (4) при $r=R_{1}$ и равны:

$$
\left\{\begin{array}{c}
\sigma_{r}\left(R_{1}\right)=q \\
\sigma_{\theta}\left(R_{1}\right)=\frac{2 \cdot p_{t} \cdot R_{t}^{2}-q \cdot\left(R_{t}^{2}+R_{1}^{2}\right)}{R_{t}^{2}-R_{1}^{2}} .
\end{array}\right.
$$

Напряжения на внешней поверхности тампонажного слоя определяются из (4) при $r=R_{\mathrm{t}}$ и равны:

$$
\left\{\begin{array}{c}
\sigma_{r}\left(R_{t}\right)=p_{t} \\
\sigma_{\theta}\left(R_{t}\right)=\frac{p_{t} \cdot\left(R_{t}^{2}+R_{1}^{2}\right)-2 \cdot q \cdot R_{1}^{2}}{R_{t}^{2}-R_{1}^{2}} .
\end{array}\right.
$$

Окружная деформация тампонажного камня для осесимметричного плоскодеформированного состояния рассчитывается как:

$$
\varepsilon_{\theta}=\frac{1}{E_{t}} \cdot\left(\sigma_{\theta} \cdot\left(1-v_{t}^{2}\right)-\left(v_{t}+v_{t}^{2}\right) \cdot \sigma_{r}\right),
$$

где $E_{t}-$ модуль линейных деформаций; $v_{t}-$ коэффициент поперечных деформаций тампонажного камня.

Подставляя выражения для напряжений (4) в (5), с учетом малости $v_{t}^{2}$, получаем радиальное смещение тампонажного камня на крепь

$$
\begin{aligned}
& u\left(R_{1}\right)=\varepsilon_{\theta}\left(R_{1}\right) \cdot R_{1}=\frac{R_{1}}{E_{t}} \cdot\left[\frac{2 \cdot p_{t} \cdot R_{t}^{2}-q \cdot\left(R_{t}^{2}+R_{1}^{2}\right)}{R_{t}^{2}-R_{1}^{2}}-\right. \\
& \left.v_{t} \cdot q\right] .
\end{aligned}
$$

Аналогично находится радиальное смещение тампонажного камня со стороны нарушенного слоя пород:

$$
u\left(R_{t}\right)=\varepsilon_{\theta}\left(R_{t}\right) \cdot R_{t}=\frac{R_{t}}{E_{t}} \cdot\left[\frac{p_{t} \cdot\left(R_{t}^{2}+R_{1}^{2}\right)-2 \cdot q \cdot R_{1}^{2}}{R_{t}^{2}-R_{1}^{2}}-\right.
$$




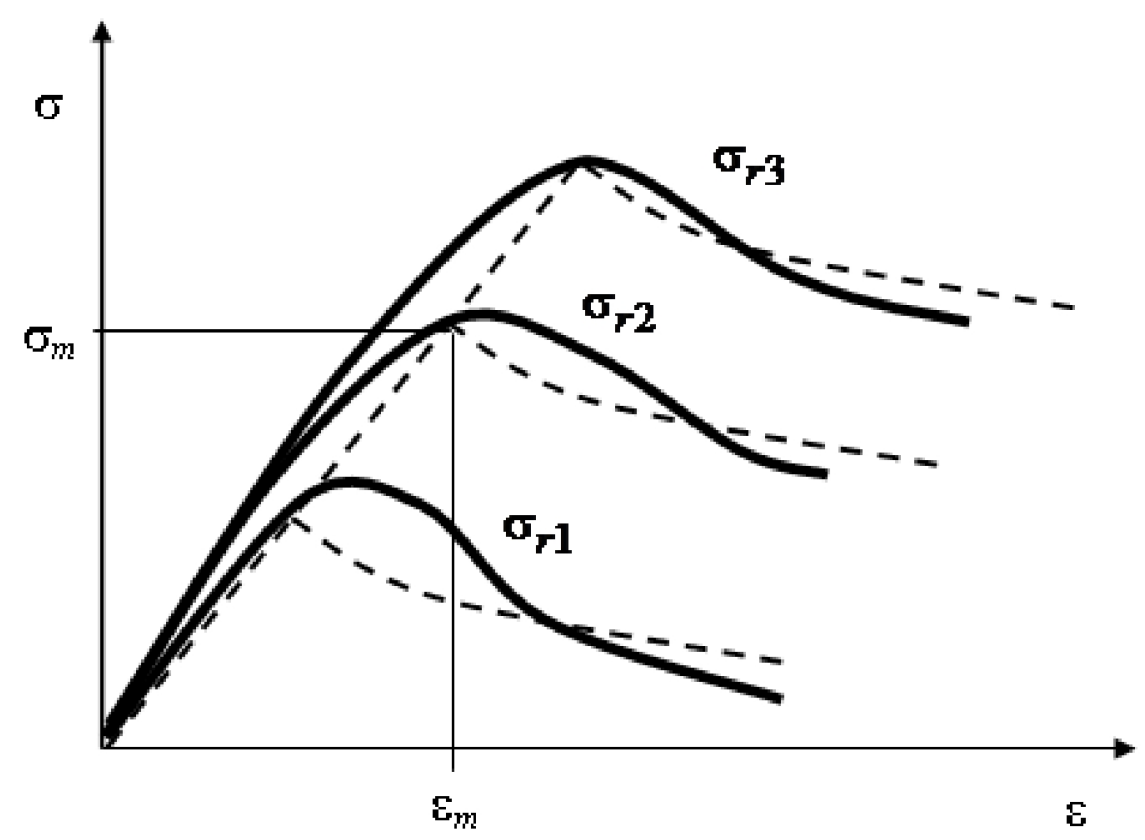

Рис. 1. Диаграммы объемного сжатия горных пород: экспериментальные кривые - сплоиные линии; теоретические кривые - пунктирные линии; $\sigma_{r 1<} \sigma_{r 2} \sigma_{r 3}$

Fig. 1. Volumetric compression diagrams of rocks: experimental curves - solid lines; theoretical curves -

$$
\text { dashed lines; } \sigma_{r 1<} \sigma_{r 2}<\sigma_{r 3}
$$

$\left.v_{t} \cdot p_{t}\right]$

Приравнивая радиальные смещения на границе крепь - тампонажный слой, из уравнений (5) и (7) получаем соотношение давлений на крепь и на тампонажный слой:

(8)

$$
p_{t}=\frac{1}{2} \cdot\left[\frac{E_{t} \cdot R \cdot\left(R_{t}^{2}-R_{1}^{2}\right)}{E_{k} \cdot h_{k} \cdot R_{t}^{2}}+\frac{R_{t}^{2}+R_{1}^{2}}{R_{t}^{2}}+v_{t} \cdot \frac{R_{t}^{2}-R_{1}^{2}}{R_{t}^{2}}\right] \cdot q,
$$

или $p_{t}=\beta \cdot q, \quad$ где $\beta=\frac{1}{2} \cdot\left[\frac{E_{t} \cdot R \cdot\left(R_{t}^{2}-R_{1}^{2}\right)}{E_{k} \cdot h_{k} \cdot R_{t}^{2}}+\right.$ $\left.\frac{R_{t}^{2}+R_{1}^{2}}{R_{t}^{2}}+v_{t} \cdot \frac{R_{t}^{2}-R_{1}^{2}}{R_{t}^{2}}\right]$.

Для определения необходимой прочности тампонажного камня используется критерий прочности горных пород при объемном сжатии [16]:

$$
\sigma_{\theta}(r)=\frac{1-v_{t}}{v_{t}} \sigma_{r}(r)+\frac{\sigma_{\text {сж }}}{1+v_{t}} .
$$

На границе тампонажного слоя с крепью минимальная прочность на сжатие тампонажного камня с учетом граничных значений напряжений определяется как:

$$
\sigma_{\mathrm{c} \aleph}^{t}\left(R_{1}\right)=\left(1+v_{t}\right) \cdot\left[\frac{(2 \cdot \beta-1) \cdot R_{t}^{2}-R_{1}^{2}}{R_{t}^{2}-R_{1}^{2}}-c_{t}\right] \cdot q .
$$

Здесь $c_{t}=\left(1-v_{t}\right) / v_{t}$.

Ширина тампонажного слоя $h_{t}=R_{t}-R_{1}$ должна быть такая, чтобы выполнялось следующее условие:

$$
\frac{(2 \cdot \beta-1) \cdot R_{t}^{2}-R_{1}^{2}}{R_{t}^{2}-R_{1}^{2}}-c_{t}>0 .
$$

При невыполнении условия (11) ограничения на прочность тампонажного камня не предусмативаются.

На границе тампонажного слоя с породой соотношения на прочность тампонажного камня имеют аналогичный вид:

$$
\sigma_{c \mathscr{C}}^{t}\left(R_{t}\right)=\left(1+v_{t}\right) \cdot\left[\frac{\beta \cdot\left(R_{t}^{2}+R_{1}^{2}\right)-2 \cdot R_{1}^{2}}{R_{t}^{2}-R_{1}^{2}}-\beta \cdot c_{t}\right] \cdot q .
$$

$$
\frac{\beta \cdot\left(R_{t}^{2}+R_{1}^{2}\right)-2 \cdot R_{1}^{2}}{R_{t}^{2}-R_{1}^{2}}-\beta \cdot c_{t}>0 .
$$

При невыполнении условия (13) ограничения на прочность тампонажного камня также не предусмативаются.

Требуемая прочность тампонажного камня равна наибольшему значению прочности из двух найденных $(10,12)$. пород

\section{3. Напряженно-деформированное состояние}

Напряженное состояние ненарушенных и частично разрушенных пород описывается уравнением (1) с граничными условиями равенства радиальных напряжений на границе с тампонажным слоем $\sigma_{r}\left(R_{t}\right)=p_{t}$ и напряженным состоянием массива горных пород $\sigma_{r}(\infty)=\sigma_{\theta}(\infty)=p$.

В зоне ненарушенных пород решение имеет вид:

$$
\left\{\begin{array}{l}
\sigma_{r}(r)=p-\frac{a}{r^{2}} \\
\sigma_{\theta}(r)=p+\frac{a}{r^{2}}
\end{array}\right.
$$

В зоне частично разрушенных пород окружные 


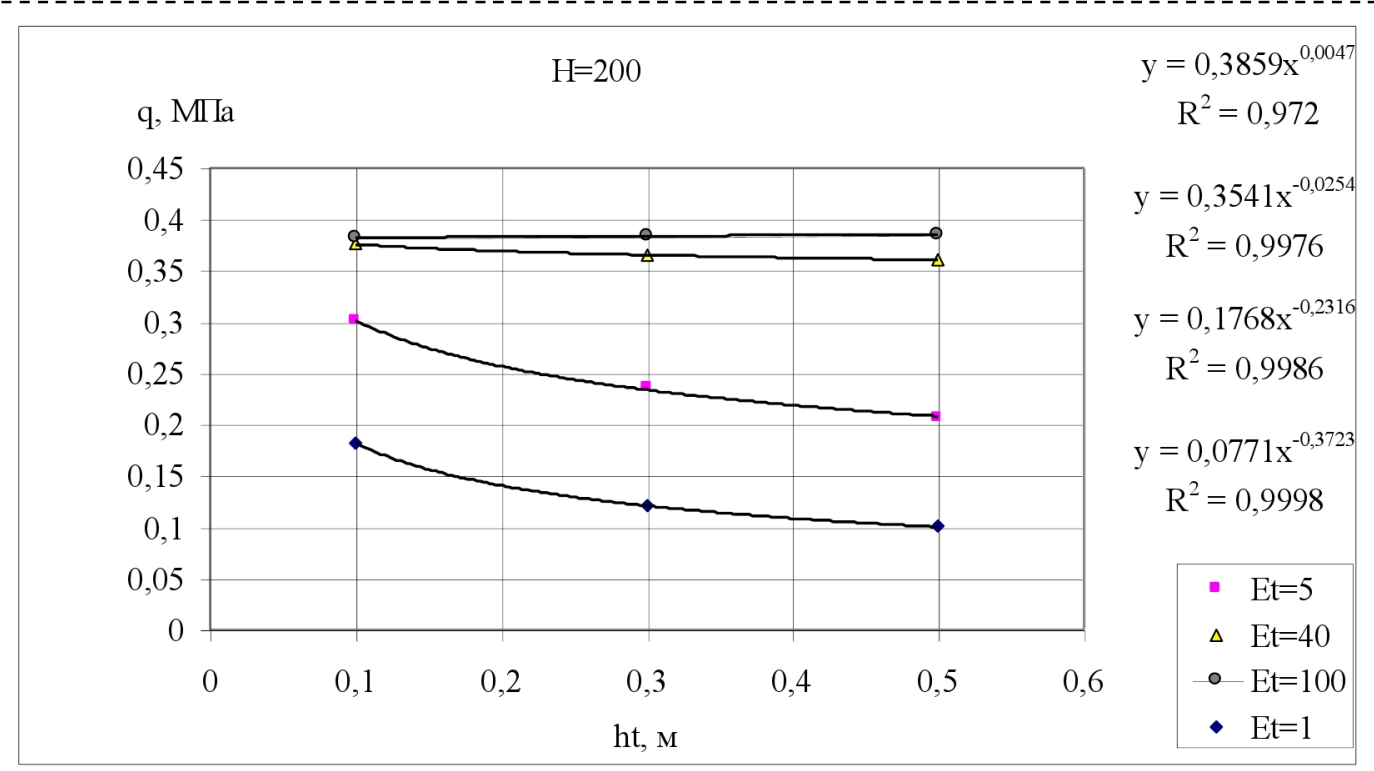

Рис.2. Закономерности изменения смещений верхняка и от иирины тампонажного слоя $h_{t}$ Fig. 2. Patterns of change of the roof girder $u$ to the width of the filling layer $h_{t}$

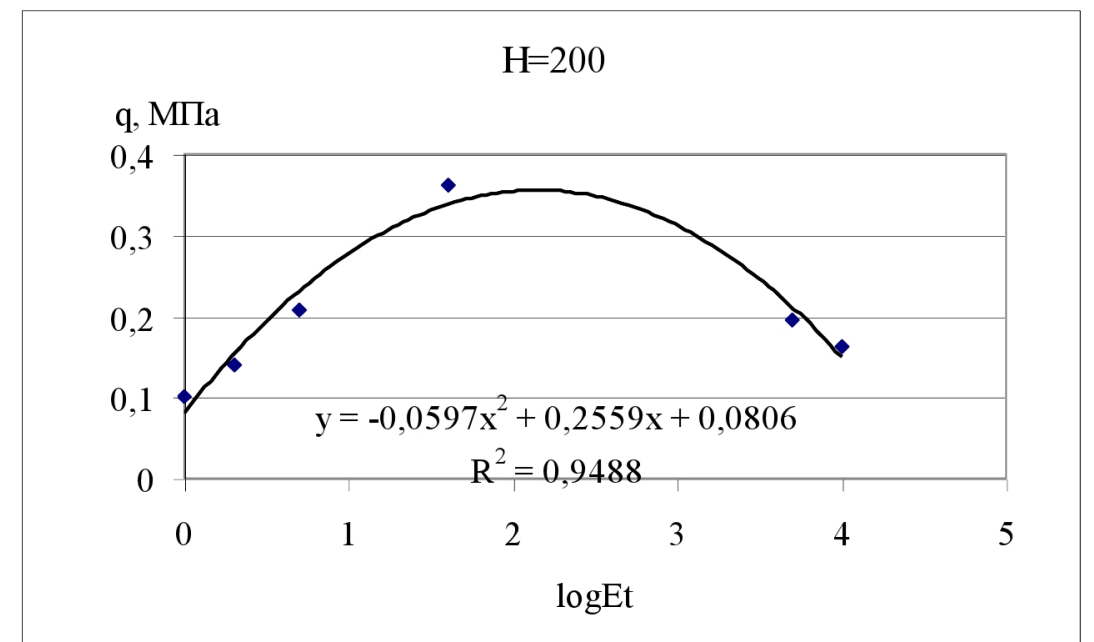

Рис. 3. Закономерность изменения давления на крепь q от модуля деформаций тампонажного слоя $E_{t}$

Fig. 3. The pattern of pressure change on the support q from the module of deformations of the filling layer $E_{t}$

и радиальные напряжения пропорциональны: $\sigma_{\theta}=$ $k \cdot \sigma_{r}$. С учетом этого решение уравнения (1) запишем как:

$$
\left\{\begin{array}{c}
\sigma_{r}(r)=p_{t} \cdot\left(\frac{r}{R_{t}}\right)^{k-1} ; \\
\sigma_{\theta}(r)=k \cdot p_{t} \cdot\left(\frac{r}{R_{t}}\right)^{k-1} .
\end{array}\right.
$$

Переход горых пород из ненарушенного состояния в частично разрушенное состояние происходит на расстоянии $R_{p}$ от центра выработки. На этом расстоянии напряженное состояние горных пород (14) переходит в напряженное состояние (15). На расстоянии $R_{p}$ от центра выработки происходит потеря прочности горных пород и переход их в запредельное состояние. На рис. 1 показаны диаграммы объемного сжатия «напряжения-деформации», описывающие переход из упругого состояния горных пород в запредельное (частично разрушенное) состояние. В точках максимума выполняется условие разрушения пород при сжатии [14]:

$$
\sigma_{\theta}\left(R_{p}\right)=\frac{1-v}{v} \sigma_{r}\left(R_{p}\right)+\frac{\sigma_{\text {Сж }}}{1+v} .
$$

С учетом непрерывности напряжений при $r=$ $R_{p}$ и критерия прочности (16) были получены значения расчетных величин.

Коэффициент, определяющий распределение напряжений в зоне частично разрушенных пород,

$k=\frac{2 c P+k_{c} \cdot \sigma_{\mathrm{cж}} /(1+v)}{2 P-k_{c} \cdot \sigma_{\mathrm{cж}} /(1+v)} ;$

Радиус зоны разрушенных пород

$R_{p}=R_{t} \cdot\left(\frac{2 P}{(k+1) p_{t}}\right)^{\frac{1}{k-1}} ;$

Коэффициент, определяющий распределение напряжений в зоне ненарушенных пород, 


$$
a=k \cdot p_{t} \cdot \frac{R_{p}^{k+1}}{R_{t}^{k-1}}-P \cdot R_{p}^{2}
$$

Максимальное окружное напряжение вмещающих пород

$$
\sigma_{m}=\frac{2 c P+k_{c} \cdot \sigma_{\mathrm{cK}} /(1+v)}{c+1},
$$

где $v$ - модуль поперечных деформаций вмещающих пород;

$$
c=\frac{1-v}{v}-\text { коэффициент, определяющий вели- }
$$

чину максимального окружного напряжения;

$\sigma_{\text {сж }}-$ прочность образца пород на одноосное сжатие;

$k_{c}$ - коэффициент структурного ослабления прочности пород на сжатие.

Теоретическая зависимость окружного напряжения от окружной деформации горных пород (см. рис. 1) представлена гиперболической кривой и имеет вид:

$$
\left\{\begin{array}{l}
\sigma_{\theta}=E \cdot \varepsilon_{\theta}, \quad \varepsilon_{\theta}<\varepsilon_{m} \\
\sigma_{\theta}=\frac{\sigma_{m}^{2}}{E \cdot \varepsilon_{\theta}}, \quad \varepsilon_{\theta}>\varepsilon_{m} .
\end{array}\right.
$$

Из (21) выражаем окружную деформацию без учета малых упругих деформаций пород, получим:

$$
\varepsilon_{\theta}=\frac{\sigma_{m}^{2}}{E \cdot \sigma_{\theta}} .
$$

Радиальные смешения частично разрушенных пород на границе с тампонажным слоем выражаются как

$$
u\left(R_{t}\right)=\varepsilon_{\theta} \cdot R_{t}=\frac{\sigma_{m}^{2} \cdot R_{t}}{E \cdot k \cdot p_{t}}
$$

Радиальные смещения со стороны тампонажного слоя имеют вид

$$
\begin{aligned}
& u\left(R_{t}\right)=\varepsilon_{\theta}\left(R_{t}\right) \cdot R_{t}=\frac{R_{t}}{E_{t}} \cdot\left[\frac{p_{t} \cdot\left(R_{t}^{2}+R_{1}^{2}\right)-2 \cdot q \cdot R_{1}^{2}}{R_{t}^{2}-R_{1}^{2}}-\right. \\
& \left.v_{t} \cdot p_{t}\right] .
\end{aligned}
$$

Приравнивая эти смещения и учитывая связь давления на тампонажный слой с давлением на крепь, после преобразований получим следующую формулу для расчета давления на крепь:

$$
\begin{aligned}
& q=\sigma_{m} \cdot \sqrt{\frac{E_{t}}{\alpha \cdot k \cdot E^{\prime}}} \\
& \text { где } \alpha=\frac{\beta^{2} \cdot\left(R_{t}^{2}+R_{1}^{2}\right)-2 \cdot \beta \cdot R_{1}^{2}}{R_{t}^{2}-R_{1}^{2}}-v_{t} \cdot \beta^{2} .
\end{aligned}
$$

С учетом упругих деформаций горных пород радиальное смещение пород на границе с тампонажным слоем принимает следующий вид:

$$
u\left(R_{t}\right)=\varepsilon_{\theta} \cdot R_{t}=\left(\frac{\sigma_{m}^{2}}{E \cdot k \cdot p_{t}} \cdot+\frac{\sigma_{m}}{E}\right) \cdot R t
$$

Приравнивая радиальные смещения со стороны тампонажного слоя (23) и радиальные смещения нарушенных пород (25), получим следующее выражение для нагрузки на крепь:

$$
q=\frac{\sigma_{m}}{2} \cdot\left(\frac{E_{t} \cdot \beta}{E \cdot \alpha}+\sqrt{\left(\frac{E_{t} \cdot \beta}{E \cdot \alpha}\right)^{2}+\frac{4 \cdot E_{t}}{E \cdot k \cdot \alpha}}\right) .
$$

В случае малой глубины проведения выработки или значительной прочности пород нарушенная зона в массиве горных пород может не образоваться. Горные породы будут оставаться в ненарушенном, упругом состоянии. Этой ситуации соответствует следующее условие, вытекающее из (17):

$$
\sigma_{\text {сж }}>\frac{2 \cdot P \cdot(1+v)}{k_{C}}
$$

По полученному значению нагрузки на крепь рассчитывается смещение верхняка в соответствии с формулой (3), проверяется прочность крепи по критерию Мора и устанавливается требуемая прочность тампонажного камня по $(11,13)$.

Полученные формулы позволили рассчитать давление на крепь в зависимости от ширины тампонажного слоя и модуля деформации тампонажного камня. На рисунках 2, 3 приведены результаты расчетов, иллюстрирующие зависимости величины давления на крепь от рассматриваемых факторов. Расчеты проведены при следующих геометрических, деформационных и прочностных параметрах: $H=200 \mathrm{M}, \quad R=2,82 \mathrm{M}, \quad h_{k}=0,14 \mathrm{M}$. $h_{t}=0,1 \div 0,5 \mathrm{M}, \quad E=10^{4} \mathrm{M \Pi a}, \quad v=0,25$, $E_{k}=2 \cdot 10^{3} \mathrm{M}$ Па, $v_{k}=0,2 ; E_{t}=1 \div 10^{4} \mathrm{M} \mathrm{a}, v_{t}=0,25$. Площадь сечения выработки $\mathrm{S}=25 \mathrm{M}^{2}$.

Приведенное выше обоснование методики расчета крепи и указанные на рис. 2, 3 закономерности изменения давления на крепь в зависимости от ширины тампонажного слоя и его модуля деформации позволяют сделать следующие выводы.

\section{Выводы.}

1. Разработана методика расчета крепи, учитывающая взаимодействие блоков «крепь - тампонажный слой - слой нарушенных горных пород массив ненарушенных горных пород».

2. Переход ненарушенных горных пород в нарушенное состояние определяется критерием прочности пород при объемном сжатии. В области нарушенных пород окружные напряжения пропорциональны радиальным напряжениям и меняются в гиперболической зависимости от окружных деформаций.

3. Наличие в закрепном пространстве тампонажного камня шириной от 0,1 до 0,5 м монотонно уменьшает давление на крепь в рассматриваемом диапазоне модуля деформации тампонажного камня от 1 МПа до 100 МПа.

4. Зависимость давления на крепь от модуля деформации тампонажного камня имеет параболический вид. При увеличении модуля деформации тампонажного камня до $10^{3} \mathrm{MПа} \mathrm{давление} \mathrm{на} \mathrm{крепь}$ возрастает. Дальнейшее увеличение жесткости тампонажного слоя приводит к снижению давления на крепь. 


\section{СПИСОК ЛИТЕРАТУРЫ}

1. Штумпф Г.Г. Горное давление в подготовительных выработках угольных шахт / Г. Г. Штумпф, П.В. Егоров, А.И. Петров, Б.В. Красильников М.: Недра, 1996. - 353 с.

2. Основные виды крепления горных выработок и методика расчета технических параметров / А.В. Ремезов, В.Г. Харитонов, Д.Н. Макшанкин, А.Ф. Брынько, В.В. Ермак. - Кемерово: Кузбассвузиздат, 2007. $-306 \mathrm{c}$.

3. Шаламанов В.А. Расчет крепей горных выработок/ В.А. Шаламанов, В.В. Першин, М.Н. Будников//Учебное пособие для студентов специальности 130406 «Шахтное и подземное строительство». - Кемерово: КузГТУ, 2011. - 86 с.

4. Карасев В.А. Разработка и обоснование параметров многозвенной спиральной крепи восстающих горных выработок. Дис. к-та техн. наук. - Кемерово, 1998. - 128 с.

5. Клыков А.Е. Дилатансия горных пород и смещения в выработке в зависимости от состояния закрепного пространства // Форум гірників - 2013: матеріали міжнар. конф., 2-5 жовтня 2013 р. - Дніпропетровськ, 2013. - Т.2. - С. 264-271.

6. Деев П.В. Вляние величины технологического зазора на напряженное состояние обделок тоннелей // П.В. Деев, А.С. Саммаль, С.В. Анциферов, Н.В. Шелепов. - Известия Тульского государственного университета. Науки о Земле, 2018..- №4, с. 287-293.

7. Черданцев С.В. Математическая модель управления процессом формирования зон нарушения сплошности вокруг выработок с винтовой крепью. / С.В. Черданцев // ГИАБ, 2006. - № 11, с. 390-395.

8. Макшанкин Д.Н. Геомеханическое обоснование крепления горных выработок металлической крепью с профилем ШП. Дис. к-та техн наук - Кемерово, 2012. -168 с.

9. Росстальной Е.Б. Лабораторные исследования влияния тампонажа закрепного пространства на несущую способность крепей методом эквивалентных материалов / Е.Б. Росстальной // Вестник КузГТУ. 2006. - №6.2. - С. 47-49

10. Тациенко В.П. Увеличение несущей способности рамной крепи путем полного заполнения закрепного пространства тампонажным материалом / В.П. Тациенко, А.С. Лисковец, М.В. Саблин // Вестник КузГТУ. - 2018. - №2. - С. 45-52.

11. Brady, B.H., \& Brown, E.T. (2013). Rock Mechanics: For Underground Mining. New York: Springer Science \& Business Media.

12. Jaeger, J.C., Cook, N.G., \& Zimmerman, R. (2009). Fundamentals of Rock Mechanics. Hoboken: John Wiley \& Sons.

13. Jing, L. (2003). A Review of Techniques, Advances and OutStanding Issues in Numerical Modelling for Rock Mechanics and Rock Engineering. International Journal of Rock Mechanics and Mining Sciences, 40(3), $283-353$.

14. Hudson, J.A., \& Harrison, J.P. (2000). Engineering Rock Mechanics: An Introduction to the Principles. New York: Elsevier.

15. Тациенко В.П. Постановка задачи расчета крепи с тампонажем закрепного простраства / В.П. Тациенко, В.А. Гоголин, И.А. Ермакова, А.С. Лисковец // Вестник КузГТУ, 2019, № 3.

16. Гоголин В.А. Деформационные и прочностные характеристики хрупких горных пород при сжатии/ В.А. Гоголин // Вестник КузГТУ, 2016, № 3. - С. 3-7.

\section{REFERENCES}

1. Shtumpf G.G. Gornoe davlenie v podgotovitel'nyh vyrabotkah ugol'nyh shaht / G. G. Shtumpf, P.V. Egorov, A.I. Petrov, B.V. Krasil'nikov M.: Nedra, 1996. - 353 p.

2. Osnovnye vidy krepleniya gornyh vyrabotok i metodika rascheta tekhnicheskih parametrov / A.V. Remezov, V.G. Haritonov, D.N. Makshankin, A.F. Bryn'ko, V.V. Ermak. - Kemerovo: Kuzbassvuzizdat, 2007. - 306 p.

3. Shalamanov V.A. Raschet krepej gornyh vyrabotok/ V.A. Shalamanov, V.V. Pershin, M.N. Budnikov //Uchebnoe posobie dlya studentov special'nosti 130406 «SHahtnoe i podzemnoe stroitel'stvo». - Kemerovo: KuzGTU, 2011. - 86 p.

4. Karasev V.A. Razrabotka i obosnovanie parametrov mnogozvennoj spiral'noj krepi vosstayushchih gornyh vyrabotok. Dis. k-ta tekhn. nauk. - Kemerovo, 1998. - 128 p.

5. Klykov A. E. Dilatansiya gornyh porod i smeshcheniya v vyrabotke v zavisimosti ot sostoyaniya zakrepnogo prostranstva // Forum girnikiv - 2013: materiali mizhnar. konf., 2-5 zhovtnya 2013 r. - Dnipropetrovs'k, 2013. T.2. - pp. 264-271.

6. Deev P.V. Vlyanie velichiny tekhnologicheskogo zazora na napryazhennoe sostoyanie obdelok tonnelej // 
P.V. Deev, A.S. Sammal', S.V. Anciferov, N.V. SHelepov. - Izvestiya Tul'skogo gosudarstvennogo universiteta. Nauki o Zemle, 2018..- №4, pp. 287-293.

7. Cherdancev S.V. Matematicheskaya model' upravleniya processom formirovaniya zon narusheniya sploshnosti vokrug vyrabotok s vintovoj krep'yu. / S.V. Cherdancev // GIAB, 2006. - № 11, pp. 390-395.

8. Makshankin D.N. Geomekhanicheskoe obosnovanie krepleniya gornyh vyrabotok metallicheskoj krep'yu s profilem SHP. Dis. k-ta tekhn nauk - Kemerovo, 2012. -168 p.

9. Rosstal'noj E.B. Laboratornye issledovaniya vliyaniya tamponazha zakrepnogo prostranstva na nesushchuyu sposobnost' krepej metodom ekvivalentnyh materialov / E.B. Rosstal'noj // Vestnik KuzGTU. - 2006. - №6.2. - C. $47-49$

10. Tacienko V.P. Uvelechenie nesushchej sposobnosti ramnoj krepi putem polnogo zapolneniya zakrepnogo prostranstva tamponazhnym materialom / V.P Tacienko., A.S. Liskovec, M.V. Sablin // Vestnik KuzGTU, 2018 - №2. - S. 45-52.

11. Brady, B.H., \& Brown, E.T. (2013). Rock Mechanics: For Underground Mining. New York: Springer Science \& Business Media.

12. Jaeger, J.C., Cook, N.G., \& Zimmerman, R. (2009). Fundamentals of Rock Mechanics. Hoboken: John Wiley \& Sons.

13. Jing, L. (2003). A Review of Techniques, Advances and OutStanding Issues in Numerical Modelling for Rock Mechanics and Rock Engineering. International Journal of Rock Mechanics and Mining Sciences, 40(3), 283-353.

14. Hudson, J.A., \& Harrison, J.P. (2000). Engineering Rock Mechanics: An Introduction to the Principles. New York: Elsevier.

15. Tacienko V.P. Postanovka zadachi rascheta krepi s tamponazhem zakrepnogo prostrastva / V.P. Tacienko, V.A. Gogolin, I.A. Ermakova, A.S. Liskovec // Vestnik KuzGTU, 2019, №3.

16. Gogolin V.A. Deformacionnye i prochnostnye harakteristiki hrupkih gornyh porod pri szhatii/ V.A. Gogolin// Vestnik KuzGTU, 2016, № 3. - pp. 3-7. 